\title{
EMBRYO CELL MEMBRANES RECONSTRUCTION BY TENSOR VOTING
}

\author{
Gaël Michelin ${ }^{\star, 1} \quad$ Léo Guignard ${ }^{\dagger, 2} \quad$ Ulla-Maj Fiuza $^{\dagger} \quad$ Grégoire Malandain $^{\star, 1}$ \\ * INRIA, 06900 Sophia Antipolis, France \\ ${ }^{\dagger}$ CRBM, UMR 5237, CNRS, Univ. Montpellier 1 \& 2, 34293 Montpellier, France
}

\begin{abstract}
Image-based studies of developing organs or embryos produce a huge quantity of data. To handle such high-throughput experimental protocols, automated computer-assisted methods are highly desirable. This article aims at designing an efficient cell segmentation method from microscopic images. The proposed approach is twofold: first, cell membranes are enhanced or extracted by the means of structure-based filters, and then perceptual grouping (i.e. tensor voting) allows to correct for segmentation gaps. To decrease the computational cost of this last step, we propose different methodologies to reduce the number of voters. Assessment on real data allows us to deduce the most efficient approach.
\end{abstract}

Index Terms - fluorescence microscopy, image segmentation, cell membrane

\section{INTRODUCTION}

The organism formation during animal or plant development is a major question in developmental biology. One challenge is to describe shape evolution as a geometrical output of gene activity $[1,2]$. Due to recent progress in microscopy, time-lapse $3 \mathrm{D}$ images of living organisms can be recorded for several hours at a high frequency that permits to monitor cell deformations and divisions [3]. This results in huge data sets that require highly robust and efficient computational image analysis tools for segmenting the cells, registering the images if necessary, and extract the cell lineages [4].

The present work focuses on the cell segmentation step. The fluorescence of cell membranes or walls makes watershed [5] a method of choice [6, 7]. However, imaging noise and artifacts as well as fluorescence defects may cause segmentation errors. While deformable models may help to produce well-shaped cells, they may be computationally expensive if a large number of cells has to be segmented. Tensor voting is a means to correct for defects in segmentation [8]. The ACME method [7] demonstrates that, combined to a membrane enhancement filter, it overperformed the watershed algorithm applied on original data, but requires an important computational effort.

We propose to follow the same methodology as in [7], with the following contributions: an alternative structure-based detection filter, and several strategies to reduce the computational cost of the tensor voting step that is directly proportional to the number of retained points. Since several approaches can be built by combination of the filters and selection strategies, we perform a quantitative assessment on real data that allows us to select the most efficient ones.

\footnotetext{
Contact: \{gael.michelin,gregoire.malandain\}@inria.fr

${ }^{1} \mathrm{GM}$ and GM are with INRIA team Morpheme.

${ }^{2} \mathrm{LG}$ is also with INRIA team Virtual Plants, UMR AGAP, Montpellier, France.
}

\section{MEMBRANE ENHANCEMENT}

\subsection{Membrane enhancement filters}

Hessian-based filters have been proposed to detect vessels in medical imaging, because of their ability to characterize line-like structures [9]. More generally, assuming that the structures of interest are bright over a dark background, and that the eigenvalues $\lambda_{i}$ of the Hessian matrix are ordered by their magnitude $\left(\left|\lambda_{3}\right| \geq\left|\lambda_{2}\right| \geq\left|\lambda_{1}\right|\right)$, a line-like structure is characterized by large (and negative) $\lambda_{3}$ and $\lambda_{2}$ and a small $\left|\lambda_{1}\right|$ (the associated eigenvector $\mathbf{v}_{1}$ gives the line direction), while a plane-like structure is characterized by a large (and negative) $\lambda_{3}$ (the associated eigenvector $\mathbf{v}_{3}$ gives the orthogonal direction to the plane) and small $\left|\lambda_{2}\right|$ and $\left|\lambda_{1}\right|$. These properties have been exploited in medical imaging to detect vessels, either by building an ad-hoc response function based on the Hessian eigenvalues [10] or by integrating an edge detection response around a vessel center candidate [11]. In other words, both approaches characterize the structure orientation thanks to the Hessian eigenvectors, while the response amplitude is based respectively on the local secondorder derivative amplitudes in Frangi's formulation and on the distant first-order derivative amplitudes in Krissian's one.

Mosaliganti and col. [7] have adapted Frangi's formulation to design a membrane enhancement filter (see Eq. 1):

$$
R_{F}(P)=\left\{\begin{array}{l}
0 \text { if } \lambda_{3} \geq 0 \\
\left(1-\mathrm{e}^{-\frac{\sum \lambda_{i}^{2}}{2 \gamma^{2}}}\right) \mathrm{e}^{-\frac{\left|\frac{\lambda_{2}}{\lambda_{3}}\right|^{2}}{2 \alpha^{2}}} \mathrm{e}^{-\frac{\left(\frac{\sqrt{\left|\lambda_{1} \lambda_{2}\right|}}{\left|\lambda_{3}\right|}\right)^{2}}{2 \beta^{2}}} \mathrm{e}^{-\frac{2 c^{2}}{\lambda_{1}^{2}}}
\end{array}\right.
$$

We propose here to adapt Krissian's approach to membranes. Although straightforward, this has not be done yet to the best of our knowledge. The response function at a point $P$ of an image $I$ is calculated by integrating an edge response at a distance $r$ to a membrane center candidate:

$$
R_{K}(P)=\left\{\begin{array}{l}
0 \text { if } \lambda_{3} \geq 0 \\
\frac{1}{2}\left(\nabla I\left(P-r \mathbf{v}_{3}\right) \cdot \mathbf{v}_{3}-\nabla I\left(P+r \mathbf{v}_{3}\right) \cdot \mathbf{v}_{3}\right)
\end{array}\right.
$$

\subsection{Extrema extraction}

The two above filters are designed so that the response is maximal at the membrane center (with respect to its orthogonal direction). Thus suppressing the non-maxima will help to keep only pertinent information while suppressing the spurious one. This is done by extracting the directional (with respect to $\mathbf{v}_{3}$ ) extrema of the response, i.e.

$$
E_{X}(P)= \begin{cases}0 & \text { if } R_{X}(P) \leq R_{X}\left(P \pm \mathbf{v}_{3}\right) \\ R_{X}(P) & \text { else }\end{cases}
$$

where $X$ is respectively $K$ for Krissian-like filter and $F$ for Frangilike one. 

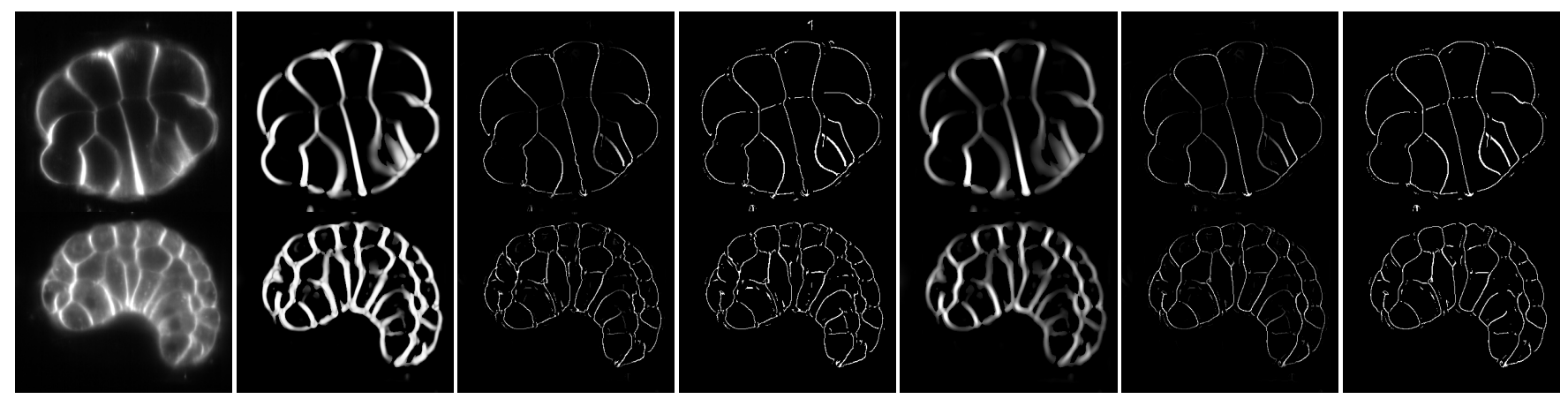

Fig. 1. First row, from left to right: a 2D cross-section of respectively the 3D image \#1 (32 cells stage), the Frangi-like response, the Frangi extrema, the thresholded extrema of Frangi extrema, the Krissian-like response, the Krissian extrema, and the thresholded extrema of Krissian extrema. The second row depicted the same for image \#91 (162 cells stage).

\subsection{Extrema thresholding}

The extrema are binarized by an hysteresis thresholding. The thresholds are chosen manually to obtain a visually good compromise between false positives and false negatives. It results a binary image $B_{X}$ where $X \in\{F, K\}$ as before.

\section{TENSOR VOTING}

The above filters are designed to enhance plane-like structures. However, they will fail to enhance them at junctions or when the signal is too weak. Perceptual grouping, by the means of tensor voting, may address the second point, while the junctions issue can be resolved by a post-process of the tensor voting result.

\subsection{Structural representation}

Structures are locally represented (at each point $P$ ) by a $2^{\text {nd }}$ order tensor, $\mathbf{T}(P)$, i.e. a $3 \times 3$ real positive, symmetric matrix. Its decomposition in eigenvalues $\kappa_{3} \geq \kappa_{2} \geq \kappa_{1} \geq 0$ and associated eigenvectors $\mathbf{e}_{i}$ allows to rewrite it as a linear combination of three generic tensors:

$$
\mathbf{T}=\underbrace{\kappa_{1} \sum_{i \in\{1,2,3\}} \mathbf{e}_{i} \mathbf{e}_{i}^{t}}_{\mathbf{T}_{B}}+\left(\kappa_{2}-\kappa_{1}\right) \underbrace{\sum_{i \in\{2,3\}} \mathbf{e}_{i} \mathbf{e}_{i}^{t}}_{\mathbf{T}_{P}}+\left(\kappa_{3}-\kappa_{2}\right) \underbrace{\mathbf{e}_{3} \mathbf{e}_{3}^{t}}_{\mathbf{T}_{S}}
$$

$\left[\mathbf{e}_{1}, \mathbf{e}_{2}, \mathbf{e}_{3}\right]$ defines a basis where

$$
\mathbf{T}_{S}=\left(\begin{array}{lll}
0 & 0 & 0 \\
0 & 0 & 0 \\
0 & 0 & 1
\end{array}\right), \mathbf{T}_{P}=\left(\begin{array}{lll}
0 & 0 & 0 \\
0 & 1 & 0 \\
0 & 0 & 1
\end{array}\right) \text { and } \mathbf{T}_{B}=\left(\begin{array}{lll}
1 & 0 & 0 \\
0 & 1 & 0 \\
0 & 0 & 1
\end{array}\right)
$$

These generic tensors are respectively named stick, plate and ball unit tensors.

- A stick tensor expresses uncertainty of data orientation in the two directions $\mathbf{e}_{1}$ and $\mathbf{e}_{2}$, it corresponds to a planar structure.

- A plate tensor expresses uncertainty in the direction $\mathbf{e}_{1}$ and corresponds to a line structure.

- A ball tensor does not express any orientation preference, it is the case in junction points.

\subsection{Tensor voting}

Tensor voting consists in building a tensor map from the votes of points $P$ or tokens, that can be points without structural information $(P \in \mathcal{B})$, or points from lines $(P \in \mathcal{P})$ or planes $(P \in \mathcal{S})$, i.e. associated to some privileged directions. For each structure type, a tensor voting field is built (see [8] for details) that aims at expanding the structures along their preferential directions according to a scale parameter $\sigma_{T}$ (figure 2). The result of tensor voting is then a tensor image $\mathbf{J}$ :

$$
\mathbf{J}_{\sigma_{T}}(M)=\sum_{\mathcal{X} \in\{\mathcal{B}, \mathcal{P}, \mathcal{S}\}} \sum_{P \in \mathcal{X}} \alpha_{\mathcal{X}}(P) \mathbf{V}_{\mathcal{X}, \sigma_{T}}\left(\mathbf{P M}, \mathbf{e}_{i}(P)\right)
$$

where $\mathbf{V}_{\mathcal{X}, \sigma_{T}}\left(\mathbf{P M}, \mathbf{e}_{i}(P)\right)$ denotes the vote of token $P$ of type $\mathcal{X} \in\{\mathcal{B}, \mathcal{P}, \mathcal{S}\}$ at point $M$ at voting scale $\sigma_{T}$, weighted by $\alpha_{\mathcal{X}}(P)$.

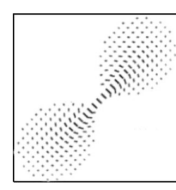

(a)

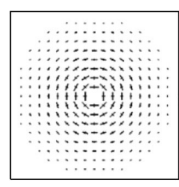

(b)

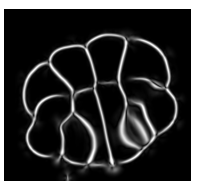

(c)

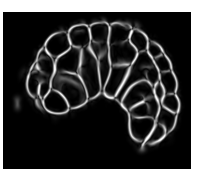

(d)
Fig. 2. Voting fields: (a) a cut of the voting field for stick component; (b) a cut of the voting field for plate component (direction $\mathbf{e}_{1}$ is normal to the page); (c) and (d) surfaceness maps $S$ after tensor voting step.

We are only interested here in plane-like structures (the membranes), hence for an image $I \in\left\{R_{X}, E_{X}, B_{X}\right\}$, we only consider stick tensor votes with

$$
\mathbf{J}_{\sigma_{T}}(M)=\sum_{P / I(P)>0} I(P) \mathbf{V}_{\mathcal{S}, \sigma_{T}}\left(\mathbf{P M}, \mathbf{v}_{3}(P)\right)
$$

where the input stick tensor is built from the eigenvector $\mathbf{v}_{3}$ (i.e. $\mathbf{e}_{3}=\mathbf{v}_{3}$ ) of the Hessian matrix, and its vote $\mathbf{V}_{\mathcal{S}, \sigma_{T}}$ is determined as:

$$
\mathbf{V}_{\mathcal{S}, \sigma_{T}}\left(\mathbf{P M}, \mathbf{v}_{3}(P)\right)=\mathrm{e}^{-\frac{s^{2}+c \kappa^{2}}{\sigma_{T}^{2}}} \mathbf{n} \mathbf{n}^{t}
$$

where $s$ is the arc length and $\kappa$ is the curvature of the arc of the osculating circle at $P$ (i.e. normal to $\mathbf{v}_{3}$ in $P$ ) which goes through 
$M . \mathbf{n}$ is the unit vector normal to the $\operatorname{arc}$ in $M$. The parameter $c$ controls the degree of vote's decay with curvature and is given by $c=-16 \log (0.1) \times\left(\sigma_{T}-1\right) \times \pi^{-2}$. Note that no votes are cast if the angle between $\mathbf{v}_{3}$ and $\mathbf{n}$ is larger than $45^{\circ}$. Since we only consider points with non-null intensity as tokens, the computational cost of tensor voting is obviously ordered from $I=R_{X}$ to $B_{X}$, as it linearly depends on the number of non-null points $P$. Please note that votes are weighted by the filter response for $I \in\left\{R_{X}, E_{X}\right\}$, the thresholded extrema value being either 0 or 1 .

\section{CELL SEGMENTATION}

From the tensor map $\mathbf{J}$, a surfaceness map $S$ is computed with $S(M)=\kappa_{3}(\mathbf{J}(M))-\kappa_{2}(\mathbf{J}(M))$ (see eq. (4)) that is subsequently used to segment the cells. For that purpose, the watershed method is used. However, this approach is known to be prone to oversegmentations and since some gaps may still exist at junctions, we design a dedicated seed extraction method, and the labeled seeds will be used as sources for the watershed instead of all minima of the $S$ image.

First, $h$-minima are extracted from $S$ [12]. Since membrane segmentation gaps form bridges between two adjacent cells, we recognize them by computing a distance map inside the extracted $h$ minima and then by extracting the $h$-maxima of the distance map. These labeled $h$-maxima are used as sources for a watershed segmentation with a regularized (i.e. convoled by a Gaussian) version of $S$, i.e. $S * G_{\sigma_{W}}$, in order to solve the junctions issue.

\section{EXPERIMENTS}

\subsection{Data}

We imaged a simple chordate organism, Phallusia mammillata, embryos. Embryo's membranes are marked by a lipophilic dye (FM464 which responds at $\sim 750 \mathrm{~nm}$ from an excitation at $595 \mathrm{~nm})$. We started the imaging session at the end of the 32 cells stage of the embryo and stoped it during its 172 cells stage. The embryo was imaged every minute from 4 different angles for 2 hours with a light-sheet microscope, the MuViSPIM [13], yielding at each timestep 4 images of around 200 slices of $1200 \times 1200$ pixels, with a pixel size of $0.26 \mu \mathrm{m}$ and a slice thickness of $1 \mu \mathrm{m}$. The 4 images were then fused to mitigate image acquisition defects due to, for example, light diffraction and/or microscope anisotropy.

One special characteristic of this setup is that the dye is slowly internalized inside the cytoplasm. It has, as impact, a degradation through time of the signal to noise ratio. This defect will allow us to have different image qualities and therefore to test two different experiment conditions. We choose for our tests the $1^{\text {st }}$ and the $91^{\text {th }}$ (after 1h30min of imaging) images from the sequence, corresponding to respectively 32 and 162 cells. While the first image may be considered as acquired under ideal imaging conditions, the second one corresponds to degrading conditions. The visual comparison of the two images (see Figure 1) depicts clearly the dye internalization.

\subsection{Computational issues}

The described method relies on a number of parameters. First, Frangi's and Krissian's filters require the computation of the image derivatives, which is achieved by convolving the image with the Gaussian derivatives. Although these filters can be embedded into a multi-scale approach to handle difference of sizes of the structures to segment, we choose to use only one scale denoted by $\sigma_{R}$ since the membranes have a homogeneous thickness. We test
$\sigma_{R} \in\{2,3,4,5,6\}$, with $r=\sigma_{R}$ in eq. 2. The extrema thresholding step is performed by fixing manually the thresholds for each extrema image in order to favour false negatives rather than false positives. The size of the tensor voting field $\mathbf{V}_{\mathcal{S}}$ is governed by an other standard deviation $\sigma_{T}$ that has to be chosen accordingly to the size of the gaps to be filled. We fix $\sigma_{T}=10$ for all the experiments. The height $h$ for the $h$-minima step is an important issue since it directly depends on the brightness of membrane structures from the surfaceness map $S$. We test $h \in\{10,15,30\}$. The $h$-maxima's height is less important to determine since it only has an influence on the size of the detected seeds. We fix the $h$-maxima parameter at 5 for the whole tests. The regularized version of $S$ used for the watershed segmentation is processed with convolutions by the derivatives of a Gaussian filter of scale $\sigma_{W}=3$ in order to remove junction gaps in $S$.

\subsection{Evaluation methodology}

The purpose of this work is to design an efficient method for cell segmentation. We want to assess the use as tokens for tensor voting of either the filter response $R_{X}$, the extracted extrema $E_{X}$, or the thresholded extracted extrema $B_{X}$ for two filters, namely the Frangilike one $(X=F)$ and the Krissian-like one $(X=K)$. This yields 6 token images to be compared, multiplied by the number of tested parameter sets. Note that using $R_{F}$ as token image is similar to the ACME method [7], thus we have a direct comparison with this approach.

The 3D images are also processed by an Fernandez's method [6]. Briefly, this is a watershed on the (regularized) original data with an ad-hoc seed/source detection. The obtained images have been manually corrected, yielding ground truth (GT) segmentation. Since we do not use the original image for the watershed, there will be unavoidable differences at the cell borders between the ground truth segmentation and the ones we obtain. In addition, we are more interested in evaluating the topological errors (i.e. the number of over-segmentations and of missed cells) than the precision of the border of the segmented cells. For these reasons, we design three measures to quantify these errors based on the comparison of the detected seeds for watershed (see section 4) against the segmented cells of the ground truth, instead of comparing the segmentations (for instance with a Dice index).

- True detections (TD) characterize a one-to-one mapping between a GT cell and a seed: the cell contains only one seed, and this seed does not intersect any other cell.

- An over-detection (OD) occurs if a cell contains more than one seed, and there will be as many over-segmentations of this cell as there are supplementary seeds (a cell containing 3 seeds counts for $2 \mathrm{OD}$ ).

- An under-detection (UD) can occur by two different ways, firstly if one cell does not contain any seed, and secondly if a seed intersects more than one cell.

\subsection{Results}

Table 1 presents the combination of tokens images and parameters that yield the largest number of True Detections (TD) together with the smallest error measures. For each combination, we present the different error measures (i.e. TD, OD and UD) but also the computational cost of the tensor voting step defined as the computational time normalized by the computational time of the ACME method (that has then a computational cost of 1 by definition). 
Table 1. Errors measures for the best combinations of token images and parameters.

\begin{tabular}{|c|c|c|c|c|c|c|c|}
\hline Img. $(\sharp$ cell $)$ & Tokens & & $h_{\min }$ & TD & OD & UD & TV cost \\
\hline \multirow{5}{*}{$\operatorname{Im} 1(32)$} & $B_{K}$ & 5 & 30 & 30 & 3 & 0 & $2.9810^{-2}$ \\
\hline & $B_{F}$ & 5 & 30 & 30 & 3 & 0 & $3.2310^{-2}$ \\
\hline & $R_{F}$ & 3 & 10 & 28 & 4 & 0 & 1.00 \\
\hline & $E_{K}$ & 4 & 10 & 28 & 4 & 0 & $12.6610^{-2}$ \\
\hline & \multicolumn{3}{|c|}{ Fernandez [6] } & 31 & 9 & 0 & \\
\hline \multirow{5}{*}{$\operatorname{Im} 2(162)$} & $B_{K}$ & 4 & 15 & 149 & 15 & 3 & $3.7410^{-2}$ \\
\hline & $E_{K}$ & 5 & 30 & $\overline{144}$ & 11 & 8 & $10.8410^{-2}$ \\
\hline & $R_{F}$ & 4 & 15 & 139 & 18 & 8 & 1.00 \\
\hline & $B_{K}$ & 3 & 15 & 136 & 29 & 2 & $3.9610^{-2}$ \\
\hline & \multicolumn{3}{|c|}{ Fernandez [6] } & 128 & 40 & 5 & \\
\hline
\end{tabular}

Results on the 162 cells image demonstrate that the structurebased approaches followed by tensor voting clearly outperform a direct watershed segment for poor quality images (because of the dye internalization, some interior cell points may have higher intensities than points of low contrast membranes).

It has to be pointed out that Mosaliganti's method [7] is in the top 4 best approaches for both test images. Moreover, all the best structure-based approaches yield comparable results in terms of segmentation quality for the high quality image (the 32 cells image). However, some differences can be noticed for the low quality image (the 162 cells image), where either the extrema or the binarized extrema of the Krissian-like filter seems to slightly outperform ACME.

More important, these two methods exhibit a significantly smaller computational cost (almost 1 or 2 order of magnitude) than the ACME method for the tensor voting step, making them the methods of choice for cell segmentation.

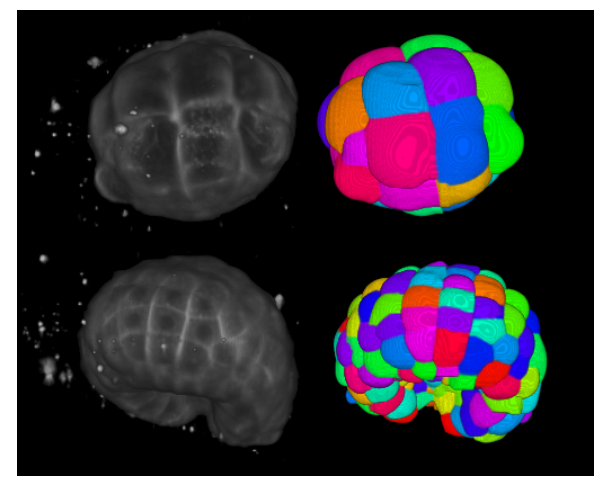

Fig. 3. From left to right: a 3D view of the 32 cells image, its segmentation (first row); the same for the 162 cells image (second row).

\section{CONCLUSION AND FUTURE WORK}

We investigated different segmentation methods, relying on a structure-based filter followed by a perceptual grouping step. The results demonstrate that: such methods outperform a direct watershed, the computational cost of tensor voting can be significantly reduced by extracting pertinent information from the structure-based filter, and a new structure-based filter (inspired from Krissian's work on vessels) slightly outperforms the Frangi-like filter. Apart of slight improvements (eg automated computation of the extrema thresholds), next steps will consist in evaluating the proposed methodol- ogy (i.e. tensor voting on thresholded Krissian's extrema) on whole $3 \mathrm{D}+\mathrm{t}$ sequences, to extract embryos cell lineages.

\section{Acknowledgements}

Lars Hufnagel (EMBL, Heidelberg, Germany) provided a valuable help for image acquisition and technical discussions. Ulla-Maj Fiuza is partially funded by the Fondation pour la Recherche Médicale.

\section{REFERENCES}

[1] E Munro, F Robin, and P Lemaire, "Cellular morphogenesis in ascidians: how to shape a simple tadpole," Curr Opin Genet Dev, vol. 16, no. 4, pp. 399-405, 2006.

[2] J Traas and O Hamant, "From genes to shape: understanding the control of morphogenesis at the shoot meristem in higher plants using systems biology," C R Biol, vol. 332, no. 11, pp. 974-85, 2009.

[3] PJ Keller, "Imaging morphogenesis: technological advances and biological insights," Science, vol. 340, no. 6137, pp. 1234168, 2013.

[4] TV Truong and W Supatto, "Toward high-content/highthroughput imaging and analysis of embryonic morphogenesis," Genesis, vol. 49, no. 7, pp. 555-69, 2011.

[5] L Vincent and P Soille, "Watersheds in digital spaces: An efficient algorithm based on immersion simulations," IEEE Trans Pattern Anal Mach Intell, vol. 13, no. 6, pp. 583-598, 1991.

[6] R Fernandez, P Das, V Mirabet, E Moscardi, J Traas, JL Verdeil, G Malandain, and C Godin, "Imaging plant growth in 4-d: robust tissue reconstruction and lineaging at cell resolution," Nat Meth, vol. 7, pp. 547-553, 2010.

[7] KR Mosaliganti, RR Noche, F Xiong, IA Swinburne, and SG Megason, "Acme: automated cell morphology extractor for comprehensive reconstruction of cell membranes," PLoS Comput Biol, vol. 8, no. 12, 2012.

[8] G Medioni, MS Lee, and CK Tang, Computational Framework for Segmentation and Grouping, Elsevier Science Inc., New York, NY, USA, 2000.

[9] C Lorenz, IC Carlsen, TM Buzug, C Fassnacht, and J Weese, "Multi-scale line segmentation with automatic estimation of width, contrast and tangential direction in 2D and 3D medical images," in CVRMed-MRCAS'97. 1997, number 1205 in LNCS, pp. 233-242, Springer.

[10] AF Frangi, WJ Niessen, KL Vincken, and MA Viergever, "Multiscale vessel enhancement filtering," in MICCAI'98. 1998, vol. 1496 of $L N C S$, pp. 130-137, Springer.

[11] K. Krissian, G. Malandain, N. Ayache, R. Vaillant, and Y. Trousset, "Model-based detection of tubular structures in 3d images," Comput Vis Image Underst, vol. 80, no. 2, pp. 130-171, 2000.

[12] P Soille, Morphological image analysis: principles and applications, Springer, 1999.

[13] U Krzic, S Gunther, TE Saunders, SJ Streichan, and L Hufnagel, "Multiview light-sheet microscope for rapid in toto imaging," Nat Methods, vol. 9, no. 7, pp. 730-3, 2012. 